\title{
Different sodium enhancements among multiple populations of Milky Way globular clusters
}

\author{
Andrés E. Piatti ${ }^{1,2}$ \\ ${ }^{1}$ Instituto Interdisciplinario de Ciencias Básicas (ICB), CONICET-UNCUYO, Padre J. Contreras 1300, M5502JMA Mendoza, \\ Argentina \\ e-mail: andres.piatti@unc.edu.ar \\ 2 Consejo Nacional de Investigaciones Científicas y Técnicas (CONICET), Godoy Cruz 2290, C1425FQB Buenos Aires, Argentina
}

Received 7 August 2020 / Accepted 25 September 2020

\begin{abstract}
We searched for clues to understand the different $\mathrm{Na}$ abundances measured in first and second generation stars of ancient Milky Way globular clusters. For that purpose, from the recent literature, we gathered the aforementioned Na abundances, orbital parameters, and structural and internal dynamical properties and ages in a homogeneous scale of 28 globular clusters. We found that the intracluster $\mathrm{Na}$ enrichment, which is measured by the difference of $\mathrm{Na}$ abundances between first and second generation stars, exhibits a trend as a function of the $\mathrm{Na}$ abundances of first generation stars, in the sense that the more Na-poor the first generation stars are, the larger the $\mathrm{Na}$ enrichment is. By using the inclinations of the globular clusters' orbits, the analyzed Na enrichments also hinted at a boundary at $\sim 0.3$ dex to differentiate globular clusters with an accreted or in situ origin, the accreted globular clusters having larger $\mathrm{Na}$ enrichments. Because relatively larger intra-cluster $\mathrm{Na}$ enhancements are seen in accreted globular clusters and small $\mathrm{Na}$ enhancements are observed in globular clusters formed in situ, although not exclusively, we speculate that the amplitude of the Na enrichment may be linked with the building block paradigm. Globular clusters at the time of formation of first and second generation stars would seem to keep a memory of this hierarchical galaxy formation process.
\end{abstract}

Key words. globular clusters: general - methods: observational

\section{Introduction}

Multiple populations is a phenomenon commonly observed in Milky Way globular clusters, which exhibit stellar populations with distinctive chemical abundance patterns (Gratton et al. 2004; Bastian \& Lardo 2018). Among the chemical elements that witness such a wide range of values, $\mathrm{Na}$ has become the flagship. This is because it has been measured in every stellar aggregate harboring multiple populations, so that intrinsic $\mathrm{Na}$ spreads have been used as observational evidence. The mechanism that triggers the enhancement of $\mathrm{Na}$, and light elements in general, is still under debate; a summary of the elements can be found in Wang et al. (2020). The most frequently discussed scenarios suggest the existence of polluters inside the clusters, which are responsible for the chemical enhancement, namely: intermediate-mass asymptotic giant branch stars, fast rotating massive main sequence stars, interacting massive binaries, and super massive main sequence stars. These polluters would enrich the intra-cluster medium in a remarkably short space of time - from nearly zero to the order of tens up to a couple hundred million years - compared with the globular clusters' ages (Oliveira et al. 2020; Saracino et al. 2020; Cassisi \& Salaris 2020).

Recently, Marino et al. (2019) compiled Na abundances for first and second generation stars in 28 Milky Way globular clusters. From the position of these stars in the chromosome map (Milone et al. 2017), they identified two types of globular clusters, called Type I and Type II globular clusters. More than $80 \%$ of the globular clusters studied by them turned out to be Type I, whose chromosome maps look much simpler than those of
Type II. With the aim of analyzing the global properties of multiple populations, they built a universal chromosome map, which revealed a tight connection with $\mathrm{Na}$ abundances. First generation stars are usually associated to those that formed at the time of the globular cluster formation, so that their chemical compositions are subject to the metallicity content of the cloud out of which the globular cluster was formed. Since some globular clusters were formed in dwarf galaxies that were later accreted by the Milky Way, while others formed in situ (Kruijssen et al. 2019), the Na abundances of their first generation stars should follow the galactic chemical enrichment at the time of galaxy formation. Indeed, $\mathrm{Na}$ abundances of first generation stars in the globular clusters studied by Marino et al. (2019) span a range of values.

As far as we are aware, there is no explanation for the level of $\mathrm{Na}$ abundances of second generation stars, which also shows a range of values among globular clusters. As such, we address this issue in this work, with the aim of providing some clues as to the origin of second generation stars. In Sect. 2, we describe the data we gathered in order to carry out our analysis, while we discuss our findings in light of a cosmological context in Sect. 3.

\section{The data}

We make use of the following four different pieces of information: the Na abundances compiled by Marino et al. (2019); dynamical properties, such as the semi-major axis, the eccentricity, the inclination of the globular clusters' orbits, and their space velocity components $\left(V_{r}, V_{\theta}, V_{\phi}\right)$, taken from Piatti (2019); the structural and internal dynamics evolutionary properties (e.g., 
half-mass relaxation times, the ratio of the cluster mass lost by tidal disruption to the total cluster mass, and the Jacobi radius), which were computed by Baumgardt et al. (2019) and Piatti et al. (2019); and the globular clusters' ages, which were homogeneously obtained by Valcin et al. (2020), using the same method, putting them in the same age scale. We note that the inclination of the globular clusters' orbits $(i)$ ranges from $0^{\circ}$ for fully prograde in-plane orbits to $90^{\circ}$ for polar orbits to $180^{\circ}$ for in-plane retrograde orbits.

Following the percepts of Forbes \& Bridges (2010), we consider retrograde motions to be the signature of globular clusters that have been accreted in the opposite rotational sense as the main bulk of the Milky Way's rotation. We note, however, that accreted globular clusters can also have prograde orbits. For this reason, Forbes \& Bridges (2010) also investigated the agemetallicity relationship as a diagnostic tool to disentangle globular clusters that accreted and formed in situ. Here, we adopt the results obtained by Piatti (2019) from the analysis of the distribution of $i$ values of 156 Milky Way globular clusters; they found a similar number of accreted globular clusters with prograde and retrograde orbits. In the subsequent analysis, we bear in mind that among the 28 globular clusters analyzed here, there could be a similar number of accreted globular clusters with prograde orbits as with retrograde ones.

As far as the completeness of the globular cluster sample is concerned, Marino et al. (2019) point out that they described the universal properties of globular clusters in the chromosome map, so that any globular cluster can be found with $\mathrm{Na}$ abundances for first and second generation stars within the quoted ranges. In this sense, the analyzed $\mathrm{Na}$ abundances are representative of those for the entire Milky Way globular cluster population. In what follows, we refer to $[\mathrm{Na} / \mathrm{Fe}]$ first and second generation stars simply by $1 \mathrm{G}$ and $2 \mathrm{G}$, respectively.

\section{Analysis and discussions}

As shown by Marino et al. (2019), Na abundance are spread among Milky Way globular clusters. Figure 1 shows that the intra-cluster enhancement in the Na abundances $(2 \mathrm{G}-1 \mathrm{G})$ is not random, but it follows a trend with $1 \mathrm{G}$, in the sense that the more $\mathrm{Na}$-poor the first generation stars, the higher the Na enhancement. In the figure, we distinguish four quadrants defined by the horizontal line at $2 \mathrm{G}-1 \mathrm{G}=0.3 \mathrm{dex}$ and the vertical line at $1 \mathrm{G}=0.0$ dex. We note that no globular cluster occupies the quadrant delimited by $2 \mathrm{G}-1 \mathrm{G}<0.3$ dex and $1 \mathrm{G}<0.0$ dex. Pal 6 has an $\mathrm{Na}$ abundance of $-0.46 \pm 0.02 \mathrm{dex}$ and is the first convincing example of a single-population globular cluster; although, its present mass $\left(\log \left(M / M_{\odot}\right)=4.83\right)$ is much higher than the lower mass limit of globular clusters with multiple populations (Villanova et al. 2013; Cassisi \& Salaris 2020). By adopting $2 \mathrm{G}-1 \mathrm{G}=0.0$ dex for Pal 6 , we find that it falls outside the range of $1 \mathrm{G}$ values for globular clusters with multiple populations. The different levels of $1 \mathrm{G}$ - analogs to field stars - shows that globular clusters formed in environments with different primordial $\mathrm{Na}$ abundances.

It has been shown that the most Na-poor limit in dwarf galaxies is lower than the $\mathrm{Na}$ abundance of Milky Way field stars, with some exceptions (Colucci et al. 2012; Ishigaki et al. 2014; Battaglia et al. 2017; Villanova et al. 2019; Salgado et al. 2019; Matsuno et al. 2019; Aguado et al. 2020). Therefore, first generation stars of globular clusters formed in accreted dwarf galaxies should mostly have $\mathrm{Na}$ abundances lower than their counterparts of globular clusters that formed in situ, which explains the range of $1 \mathrm{G}$ values seen in Fig. 1. The $\mathrm{Na}$ abundance of field stars that

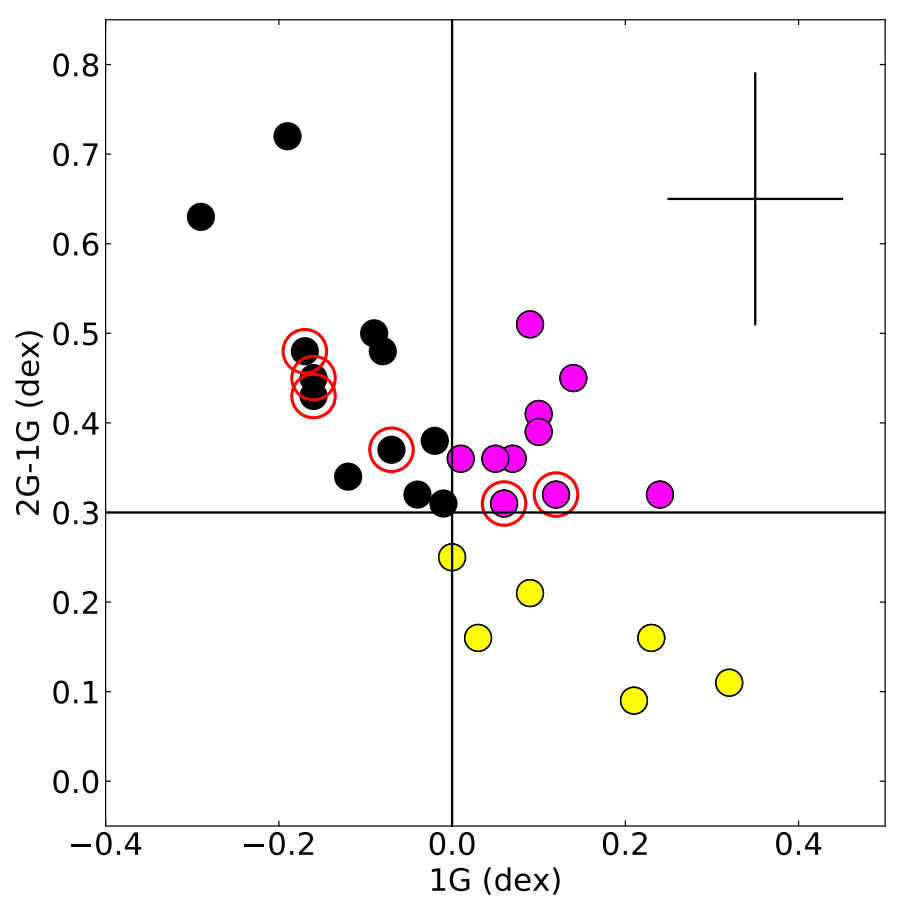

Fig. 1. Difference of $\mathrm{Na}$ abundances between first and second generation stars versus that for the first generation stars. Typical error bars are included. Black, magenta, and yellow circles represent the globular clusters located in three different quadrants, defined by $2 \mathrm{G}-$ $1 \mathrm{G}=0.3 \mathrm{dex}$ and $1 \mathrm{G}=0.0 \mathrm{dex}$. Large open red circles represent Type II globular clusters.

formed in situ is nearly 0.0 dex (see, e.g., Hill et al. 2019), so that we can assume $1 \mathrm{G}=0.0 \mathrm{dex}$ as a first guess for a representative boundary to differentiate between globular clusters that were accreted from those that formed in situ. We note, however, that there could be accreted globular clusters with $\mathrm{Na}$ abundances of first generation stars similar to that of globular clusters that formed in situ.

According to the building block paradigm (White \& Rees 1978; Font et al. 2011), dwarf galaxies that formed in an earlier Universe are expected to be older and more chemically deficient than galaxies that formed from the assembly of those primordial dwarfs. Figure 2 shows that the $1 \mathrm{G}$ values, which refer to the most Na-poor values of the galaxies where the globular clusters formed, hints at an age- $\mathrm{Na}$ abundance relationship in agreement with the mentioned galaxy formation scenario. As can be seen, globular clusters younger than $12 \mathrm{Gyr}$ are more Na-rich than 0.0 dex, while the most Na-poor globular clusters $(1 \mathrm{G}<0.0 \mathrm{dex})$ are among the oldest ones. Nevertheless, there are old globular clusters with Na-rich values $(1 \mathrm{G}>0.0 \mathrm{dex})$, which somehow reveals that the $\mathrm{Na}$ enrichment was more intense during the first $\sim 2$ Gyr. We point out that, because of the relative short space of time between the formation of first and second generation stars, second generation stars in accreted globular clusters have formed before the globular clusters that were accreted to the Milky Way. This means that the difference between $2 \mathrm{G}-1 \mathrm{G}$ is a measure of the intra-cluster $\mathrm{Na}$ enhancement at the time of the globular cluster formation.

We played with the different globular cluster parameters mentioned in Sect. 2 and found that the inclination of the globular clusters' orbits can help to recognize globular clusters that were accreted from versus those that formed in situ. The remaining astrophysical parameters do not show any clear correlation 


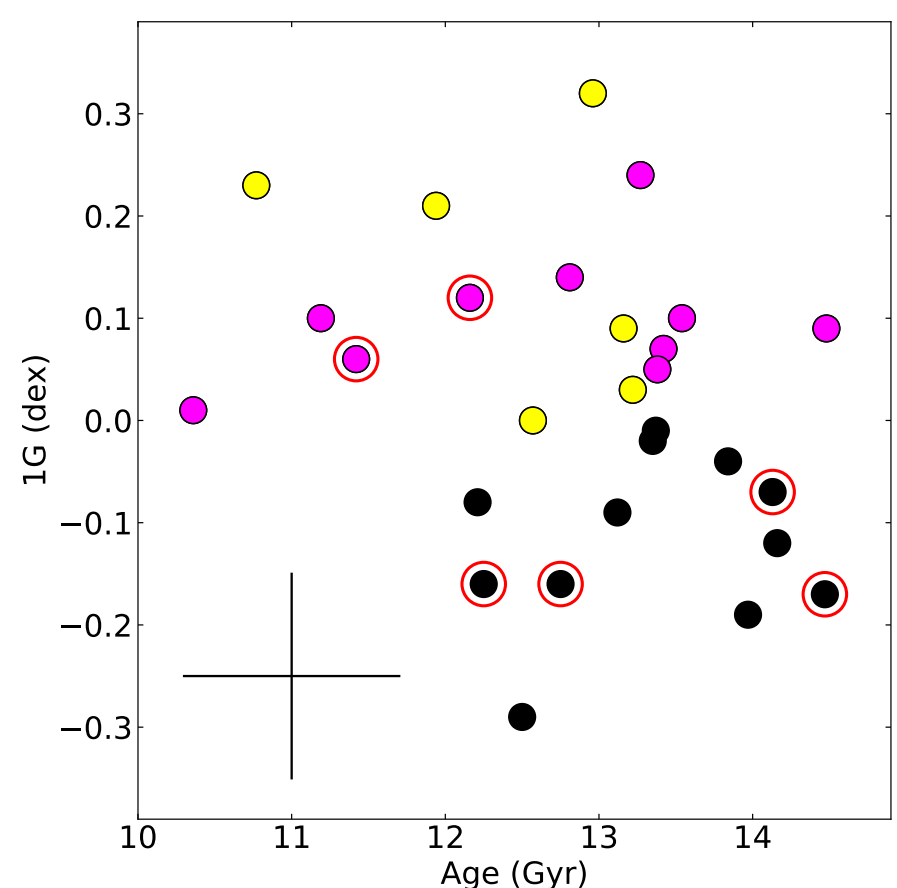

Fig. 2. Na abundance of first generation stars versus the globular clusters' ages. Typical error bars are included. Symbols are the same as in Fig. 1.

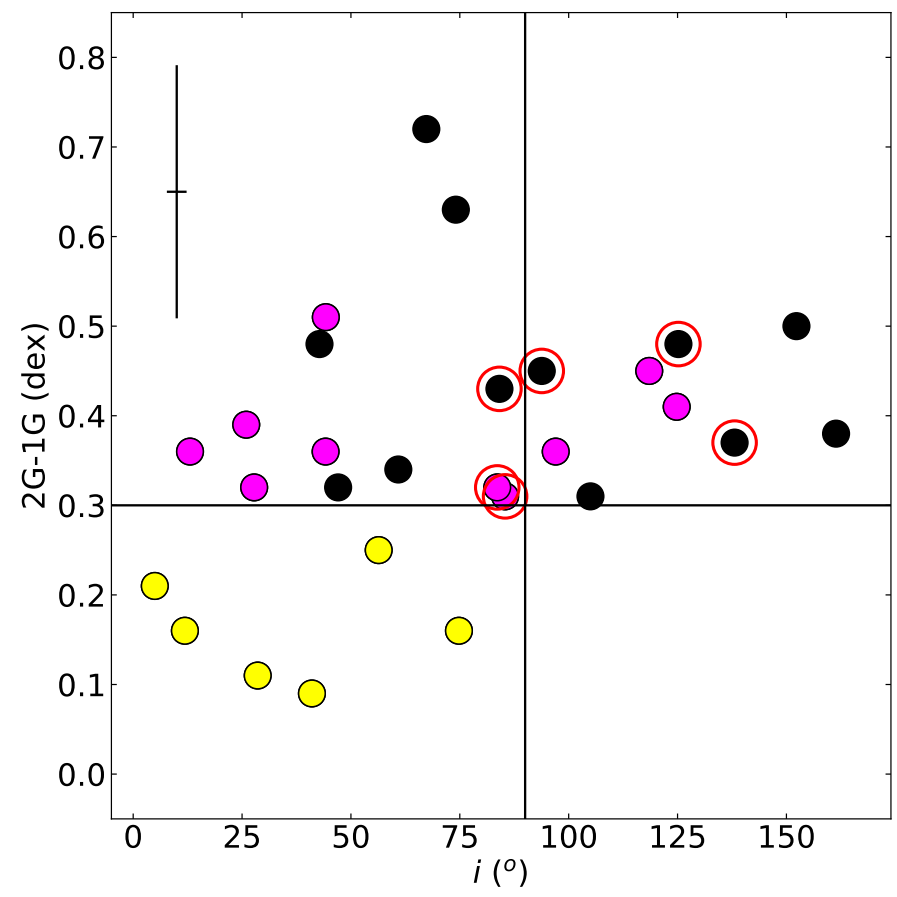

Fig. 3. Difference of $\mathrm{Na}$ abundances between first and second generation stars versus the inclination of the globular clusters' orbits. Typical error bars are included. Symbols are the same as in Fig. 1.

with $1 \mathrm{G}$ nor with $2 \mathrm{G}-1 \mathrm{G}$ (see Appendix A). Figure 3 shows that globular clusters with retrograde orbits $\left(i>90^{\circ}\right)$, and hence with an accreted origin, have $2 \mathrm{G}-1 \mathrm{G}>0.3$ dex. These globular clusters are Na-poor $(1 \mathrm{G}<0.0$ dex, black circles in Fig. 1) and older than 12 Gyr (see Fig. 2), or old and Na-rich. In either case, their orbital inclinations, ages, and $\mathrm{Na}$ abundances agree well with having formed in accreted dwarf galaxies. Therefore, we adopt

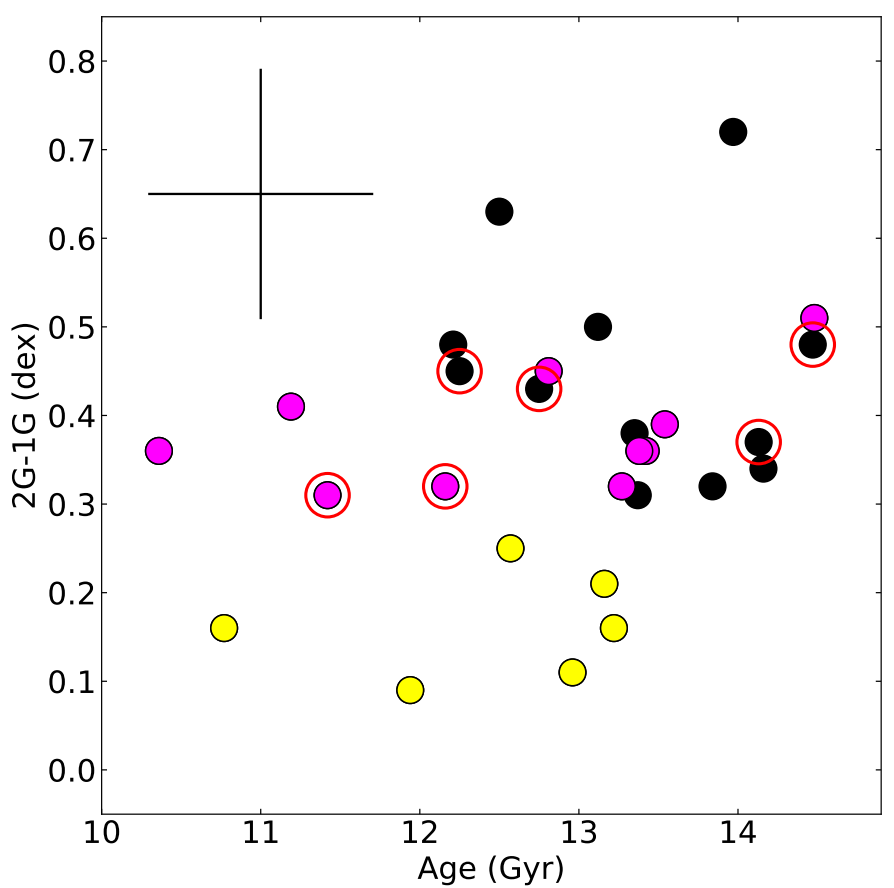

Fig. 4. Difference of Na abundances between first and second generation stars versus the globular clusters' ages. Typical error bars are included. Symbols are the same as in Fig. 1.

$2 \mathrm{G}-1 \mathrm{G}=0.3$ dex as a boundary to differentiate between globular clusters with accreted or in situ origins. According to Piatti (2019), half of the accreted globular clusters could have prograde orbital motions $\left(i<90^{\circ}\right)$. In this sense, the older and more Na-poor globular clusters (black circles) in the top-left quadrant would correspond to accreted globular clusters (see also Fig. 1), as well as some of the magenta ones. We note that it is not possible to assess a globular cluster's origin using Fig. 3 for those with $1 \mathrm{G}>0.0$ dex, $2 \mathrm{G}-1 \mathrm{G}>0.3 \mathrm{dex}$, and ages older than $12 \mathrm{Gyr}$. Figure 1 can also be used as a complementary diagnostic diagram. The trend shown in the figure can now be interpreted in terms of the frequently referred to cosmological hierarchy of galaxy formation. The oldest globular clusters formed in primordial dwarf galaxies with very deficient $\mathrm{Na}$ abundances, while those that formed in the Milky Way had more Na-rich values.

The globular clusters seen in Fig. 1 not only have different $1 \mathrm{G}$ values, but also $\mathrm{Na}$ enhancements $(2 \mathrm{G}-1 \mathrm{G})$. A possible interpretation for such a difference between these two groups of globular clusters can be drawn from Fig. 4, where the $\mathrm{Na}$ enhancements are plotted as a function of the globular clusters' ages. Figure 4 reveals some broad correlations, in the sense that the older globular clusters are, the higher the intra-cluster $\mathrm{Na}$ enhancement is. As discussed above, most of the black and some magenta circles could represent the globular clusters that formed in dwarf galaxies, which were later involved in the assembly of the Milky Way. Therefore, some globular clusters that formed inside these first galaxies could have experienced more vigorous enhancement processes during their formation, which resulted in a wider range of $\mathrm{Na}$ abundances, as compared with most of the globular clusters that formed in situ. In other words, the $\mathrm{Na}$ enrichment inside globular clusters would seem to have been more efficient in the early Universe than at the time of formation of globular clusters in the Milky Way. This picture leads us to speculate about some kind of loss of strength or deceleration of the $\mathrm{Na}$ enrichment process inside the globular clusters. If 
Table 1. Origin of Milky Way globular clusters.

\begin{tabular}{lccc}
\hline \hline Star cluster & Progenitor & Adopted & orbit's inclination $\left(^{\circ}\right)$ \\
\hline NGC 104 & Main disk (4) & In situ & 27.8 \\
NGC 288 & Gaia-Enceladus (1, 6) & Large satellite & 124.8 \\
NGC 362 & Gaia-Enceladus (1, 6), Kraken (2), Gaia-Sausage (3) & Large satellite & 85.4 \\
NGC 1851 & Gaia-Enceladus (1, 6), Canis Major (2); Gaia-Sausage (3) & Large satellite & 93.8 \\
NGC2808 & Canis Major (2), Gaia-Sausage (3), Gaia-Enceladus (4, 6) & Large satellite & 13.1 \\
NGC 3201 & Kraken (2), Sequoia (5, 6), Gaia-Enceladus/Sequoia (4) & Large satellite & 152.3 \\
NGC 4590 & Canis Major (2), Helmi streams (4, 6) & Large satellite & 41.0 \\
NGC 4833 & Gaia-Enceladus (1) & Large satellite & 44.2 \\
NGC 5024 & Helmi streams (4, 6) & Large satellite & 74.8 \\
NGC 5139 & Gaia-Enceladus (1), Kraken (2), Sequoia (5, 6) & Large satellite & 138.1 \\
NGC 5272 & Kraken (2), Helmi streams (4,6) & Large satellite & 56.4 \\
NGC 5286 & Gaia-Enceladus (1, 6), Canis Major (2), Gaia-Sausage (3) & Large satellite & 125.2 \\
NGC 5904 & Kraken (2), Helmi streams (4,6)/Gaia-Enceladus (4) & Large satellite & 74.1 \\
NGC 5986 & Low-energy (4), Koala (6) & Small satellite & 60.9 \\
NGC 6093 & Low-energy (4), Koala (6) & Small satellite & 97.0 \\
NGC 6121 & Los-energy (4), Kraken (2) & Small satellite & 5.0 \\
NGC 6205 & Gaia-Enceladus (1,6), Canis Major (2) & Large satellite & 105.0 \\
NGC 6254 & Low-energy (4), Koala (6) & Small satellite & 42.8 \\
NGC 6362 & Main disk (4) & In situ & 44.2 \\
NGC 6397 & Main disk (4) & In situ & 47.1 \\
NGC 6535 & Sequoia (5, 6)/low-energy/Sequoia (4) & Large satellite & 161.4 \\
NGC 6715 & Saggitarius (2,6) & Large satellite & 83.6 \\
NGC 6752 & Kraken (2), Main disk (4) & In situ & 26.0 \\
NGC 6809 & Low-energy (4) & Small satellite & 67.3 \\
NGC 6838 & Main disk (4) & In situ & 11.9 \\
NGC 7078 & Canis Major (2), Main disk (4) & In situ & 28.6 \\
NGC 7089 & Gaia-Enceladus (1, 6), Kraken (2), Gaia-Sausage (3) & Large satellite & 84.1 \\
NGC 7099 & Gaia-Enceladus (1, 6) & Large satellite & 118.5 \\
\hline
\end{tabular}

References. (1) Helmi et al. (2018); (2) Kruijssen et al. (2019); (3) Myeong et al. (2018); (4) Massari et al. (2019); (5) Myeong et al. (2019); (6) Forbes (2020).

the second generation of stars were the result of the interaction of binary stars, the ejecta of asymptotic giant branch stars, fast rotating main sequence stars, or stellar mergers, among others (Wang et al. 2020), the above findings would imply that those processes would have lost effectiveness in producing Na-rich stars. The lack of detection of multiple populations in star clusters younger than $2 \mathrm{Gyr}$ and less massive than $\sim 10^{5} M_{\odot}$ (see Fig. 32 in Cassisi \& Salaris 2020) could be a sign of a loss of $\mathrm{Na}$ enhancement. Indeed, cluster mass would not seem to drive multiple populations as very old globular clusters that are less massive than $10^{5} M_{\odot}$ harbor multiple populations.

Type II globular clusters are characterized by having additional sequences in the chromosome map in comparison with Type I globular clusters. These additional sequences consist of red giant branch stars, which can have $\mathrm{Na}$ abundances similar or higher than the so-called second generation stars. For this reason, the total $\mathrm{Na}$ enhancement in these globular clusters could be a little higher than the respective $(2 \mathrm{G}-1 \mathrm{G})$ value. Type II globular clusters are also enhanced in metallicity and, in some cases, $s$-process elements. These characteristics refer to a relatively faster nucleosynthesis process than the one that could take place in Type I globular clusters, which in turn match the features of accreted globular clusters found in our diagnostic diagrams. As can be seen, all Type II globular clusters in our sample have $\mathrm{Na}$ enhancements higher than 0.3 (see Fig. 1), and half of them have retrograde orbital motions (see Fig. 3).

We note that $\mathrm{Na}$ enhancement has been the most frequent observational evidence to assess the existence of multiple populations. Here, we show that the amplitude of such an $\mathrm{Na}$ enrichment could be linked with the powerful strength deployed in the early Universe, which became a more quiescent nucleosynthesis activity soon after. Although multiple populations seem to arise from within the star clusters as a result of intracluster processes (e.g., interactions between stars), the star clusters at the time of formation of first and second generation stars would seem to keep a memory of that cosmological vitality. Therefore, host galaxies would play a role in the existence of the multiple population phenomenon. We note that Milone et al. (2020) did not find any significant differences in the multiple populations between star clusters associated with different progenitors (see also Saracino et al. 2020). However, there has been a number of numerical and observational works attempting to describe the formation of globular clusters with multiple populations that are in very good agreement with some aspects of the intra-cluster $\mathrm{Na}$ enrichment scenario suggested in this work (see, e.g., Bekki 2006; Carretta et al. 2010; Maxwell et al. 2014; Battaglia et al. 2017; Santistevan et al. 2020).

We distinguish globular clusters with an accreted origin or those that formed in situ based on a combination of their kinematics (prograde versus retrograde orbits) and the $\mathrm{Na}$ abundandes of first and second generation stars. Recently, a fairly substantial piece of work has dealt with the classification of Milky Way globular clusters, according to the progenitors to which they could be associated. Table 1 shows such a compilation of possible progenitors. The last column lists the status of the globular clusters' origins adopted in this work. Piatti (2019) 


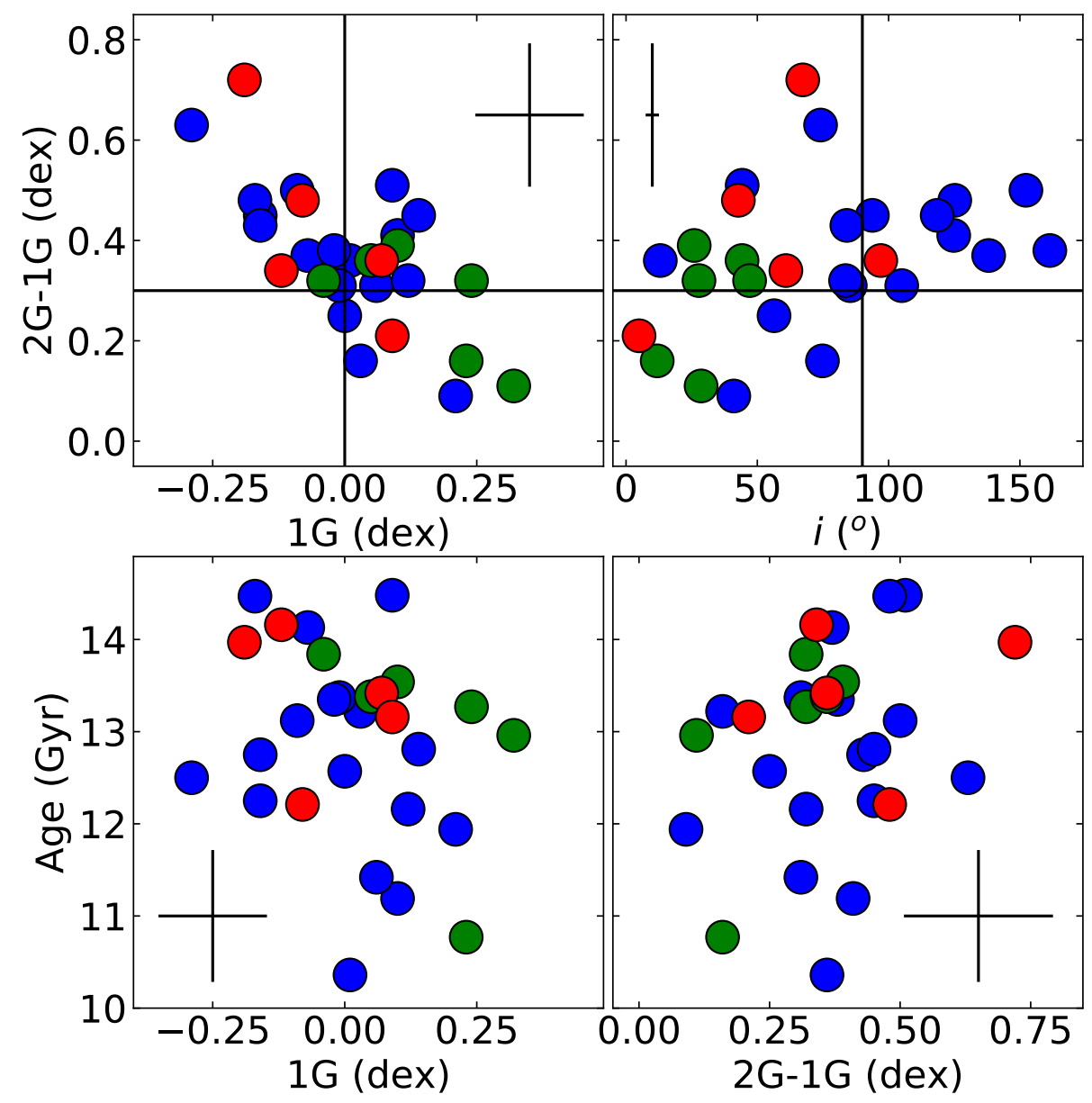

Fig. 5. Relations between $\mathrm{Na}$ abundances of first and second generation stars, the inclination of the globular clusters' orbits $(i)$, and their ages. Globular clusters, with an accreted origin from known large and small satellites, and those that formed in situ are represented by blue, red, and green filled circles, respectively. Typical error bars are included. extensively discusses the different classifications of Table 1, showing that there is some overlap in the list of globular clusters associated to each progenitor. Another aspect worth mentioning is that we find globular clusters with prograde and retrograde orbits among those associated to a particular progenitor. This means that the selection of globular clusters associated to accreted dwarf galaxies based on their angular momentum, their energies, or on age-metallicity relationships, separately, is not sufficient selection criteria.These astrophysical properties, in addition to other properties, would seem to be needed. The ratio of accreted to in situ globular clusters is also different in those studies, so that it is still an open question whether the accreted globular clusters have been shaped by minor mergers or by one major merger event. Despise the above constraints, it is still useful to explore whether the results found in this work can be globally tracked considering the adopted progenitors of Table 1. Figure 5 depicts the relationships between $1 \mathrm{G}$ and $2 \mathrm{G}-1 \mathrm{G}$ with $i$ and the globular clusters' ages as in Figs. 1-4. As can be seen in these figures, there is a broad correspondence that supports the present outcomes, in the sense that larger $\mathrm{Na}$ enhancements are seen in globular clusters associated to accreted satellites.

Tolstoy et al. (2009) showed that the $[\mathrm{Na} / \mathrm{Fe}]$ ratio varies as a function of $[\mathrm{Fe} / \mathrm{H}]$ even in Milky Way field stars, and it can be significantly subsolar at moderately low metallicities. Carretta et al. (2009) showed that the minimum $[\mathrm{Na} / \mathrm{Fe}]$ in globular clusters follows this trend quite well. With the updated compilation of $[\mathrm{Fe} / \mathrm{H}]$ abundances for first and second generations stars by Marino et al. (2019), we built Fig. 6 (top panels), which shows that such a correlation is confirmed for globular clusters with a large satellite progenitor to some extent. Globular clusters that formed in situ would not seem to exhibit a similar behavior. Likewise, the difference of $[\mathrm{Fe} / \mathrm{H}]$ values between the results of first and second generation stars is independent of $\mathrm{Na}$ enhancement (top-right panel of Fig. 6). Variations in $[\mathrm{Na} / \mathrm{Fe}]$ in field stars are usually correlated with differences in the abundances of other elements, notably the alpha-elements $(\mathrm{Mg}, \mathrm{Si}$, etc.) (see, e.g., Horta et al. 2020). Here, we probe such a trend with the $\mathrm{Mg}$ and $\mathrm{Si}$ abundances available for a subsample of the studied globular clusters (Marino et al. 2019). As can be seen in Fig. 6 (middle and bottom panels), it would seem that this is not the case for the present Milky Way globular cluster sample.

For completeness purposes, we examined the age-metallicity relationship of the studied globular clusters. We include the entire globular cluster sample in the same plot, although different age-metallicity relationships have been invoked in order to recognize globular clusters associated to different progenitors (Kruijssen et al. 2019; Massari et al. 2019; Forbes 2020). The resulting age-metallicity relationship is shown in Fig 7, where the progenitor status of Table 1 was considered. Figure 7 shows a combination of the outcomes illustrated in Figs. 1, 2, and 6 (top panels). It reveals that the most Na-poor globular clusters $(1 \mathrm{G}<0.0$ dex, see Fig. 2$)$ are older than $\sim 12 \mathrm{Gyr}$, and most of them have been assigned an accreted origin (see Fig. 5, bottomleft panel). We note that most of the globular clusters that formed in situ, regardless of their ages, are among those with Na-rich values ( $1 \mathrm{G}>0.0 \mathrm{dex}$, see Fig. 5), which is a feature that is also seen in younger globular clusters $(\lesssim 12 \mathrm{Gyr})$ with an accreted origin. Globular clusters that formed in situ span the whole age range and do not follow a tight age-metallicity relationship (see also Figs. 5 and 6 top panels). The bottom panel of Fig. 7 shows 

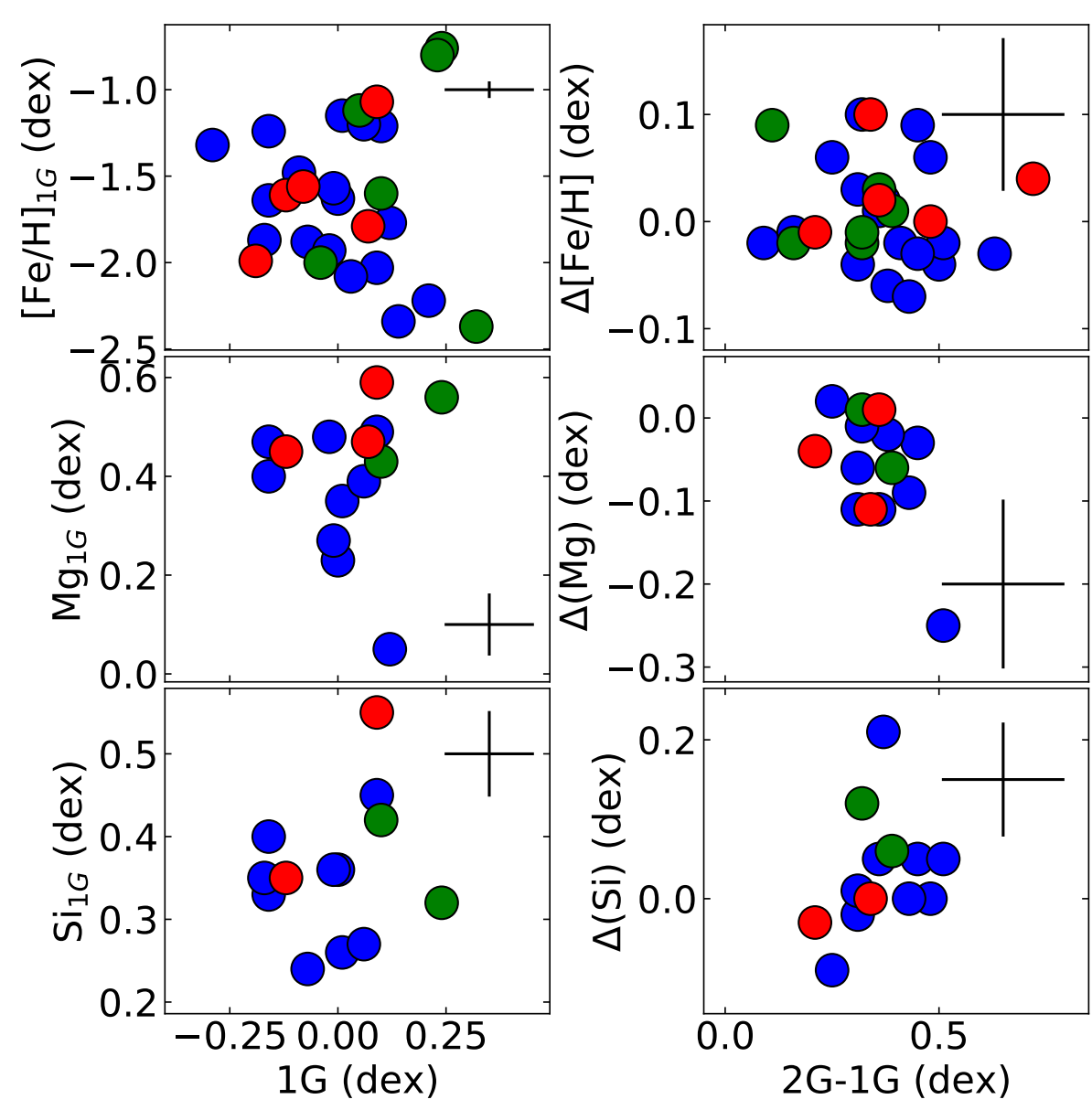

Fig. 6. Relations between $\mathrm{Fe}, \mathrm{Mg}, \mathrm{Si}$, and $\mathrm{Na}$ abundances of first and second generation stars. Symbols are the same as in Fig. 5. Typical error bars are included. We point out, as a caveat, the small number of in situ globular clusters in the studied sample. Likewise, we refer to Nissen \& Schuster (2010) where the reader might find some support for the idea that $[\mathrm{Na} / \mathrm{Fe}]$ is related to an accretion origin.

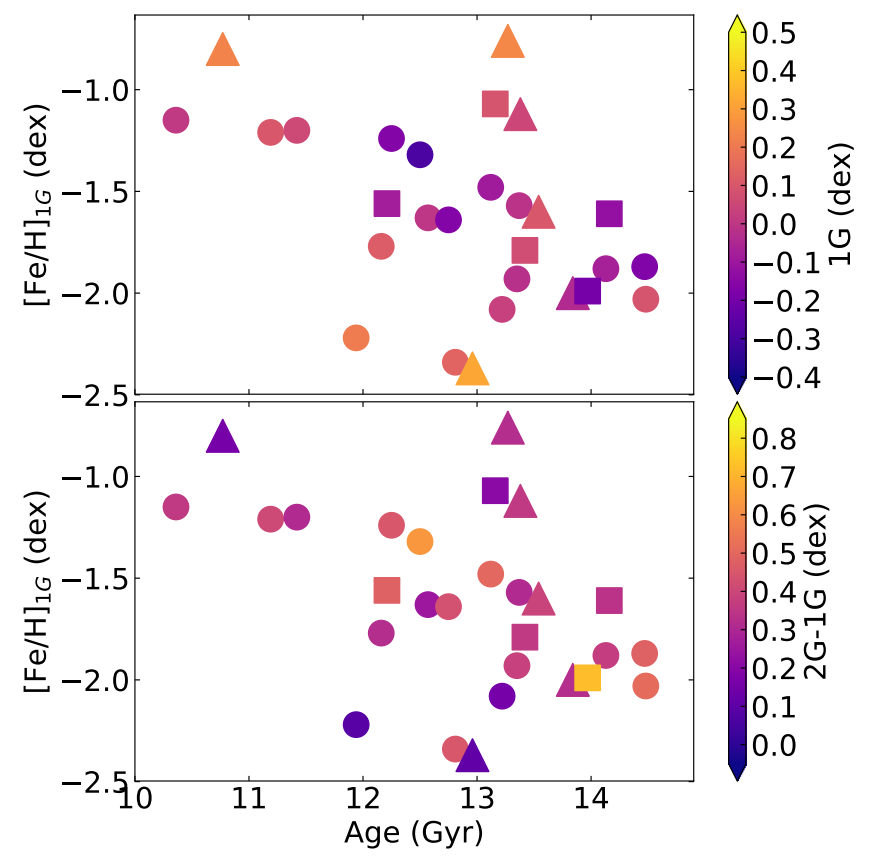

Fig. 7. Age-metallicity relationship for the studied globular cluster sample. Filled circles, squares, and triangles represent the globular clusters associated to large and small accreted satellites and those that formed in situ, respectively. Color-coded symbols represent $1 \mathrm{G}$ (top panel) and 2G-1G (bottom panel) values.

that globular clusters that formed in situ show low Na enhancements in general.
Acknowledgements. I thank the referee for the thorough reading of the manuscript and timely suggestions to improve it.

\section{References}

Aguado, D. S., Myeong, G. C., Belokurov, V., et al. 2020, MNRAS, submitted [arXiv:2007.11003]

Bastian, N., \& Lardo, C. 2018, ARA\&A, 56, 83

Battaglia, G., North, P., Jablonka, P., et al. 2017, A\&A, 608, A145

Baumgardt, H., Hilker, M., Sollima, A., \& Bellini, A. 2019, MNRAS, 482, 5138

Bekki, K. 2006, MNRAS, 367, L24

Carretta, E., Bragaglia, A., Gratton, R., \& Lucatello, S. 2009, A\&A, 505, 139

Carretta, E., Bragaglia, A., Gratton, R. G., et al. 2010, A\&A, 516, A55

Cassisi, S., \& Salaris, M. 2020, A\&ARv, 28, 5

Colucci, J. E., Bernstein, R. A., Cameron, S. A., \& McWilliam, A. 2012, ApJ, 746, 29

Font, A. S., McCarthy, I. G., Crain, R. A., et al. 2011, MNRAS, 416, 2802

Forbes, D. A. 2020, MNRAS, 493, 847

Forbes, D. A., \& Bridges, T. 2010, MNRAS, 404, 1203

Gratton, R., Sneden, C., \& Carretta, E. 2004, ARA\&A, 42, 385

Helmi, A., Babusiaux, C., Koppelman, H. H., et al. 2018, Nature, 563, 85

Hill, V., Skúladóttir, Á., Tolstoy, E., et al. 2019, A\&A, 626, A15

Horta, D., Schiavon, R. P., Mackereth, J. T., et al. 2020, MNRAS, 493, 3363

Ishigaki, M. N., Aoki, W., Arimoto, N., \& Okamoto, S. 2014, A\&A, 562, A146

Kruijssen, J. M. D., Pfeffer, J. L., Reina-Campos, M., Crain, R. A., \& Bastian, N. 2019, MNRAS, 486, 3180

Marino, A. F., Milone, A. P., Renzini, A., et al. 2019, MNRAS, 487, 3815

Massari, D., Koppelman, H. H., \& Helmi, A. 2019, A\&A, 630, L4

Matsuno, T., Aoki, W., \& Suda, T. 2019, ApJ, 874, L35

Maxwell, A. J., Wadsley, J., Couchman, H. M. P., \& Sills, A. 2014, MNRAS, 439, 2043

Milone, A. P., Piotto, G., Renzini, A., et al. 2017, MNRAS, 464, 3636

Milone, A. P., Marino, A. F., Da Costa, G. S., et al. 2020, MNRAS, 491, 515

Myeong, G. C., Evans, N. W., Belokurov, V., Sanders, J. L., \& Koposov, S. E. 2018, ApJ, 863, L28

Myeong, G. C., Vasiliev, E., Iorio, G., Evans, N. W., \& Belokurov, V. 2019, MNRAS, 488, 1235 
Nissen, P. E., \& Schuster, W. J. 2010, A\&A, 511, L10

Oliveira, R. A. P., Souza, S. O., Kerber, L. O., et al. 2020, ApJ, 891, 37

Piatti, A. E. 2019, ApJ, 882, 98

Piatti, A. E., Webb, J. J., \& Carlberg, R. G. 2019, MNRAS, 489, 4367

Salgado, C., Da Costa, G. S., Norris, J. E., \& Yong, D. 2019, MNRAS, 484, 3093

Santistevan, I. B., Wetzel, A., El-Badry, K., et al. 2020, MNRAS, 497, 747

Saracino, S., Martocchia, S., Bastian, N., et al. 2020, MNRAS, 493, 6060

Tolstoy, E., Hill, V., \& Tosi, M. 2009, ARA\&A, 47, 371

Valcin, D., Bernal, J. L., Jimenez, R., Verde, L., \& Wandelt, B. D. 2020, ArXiv e-prints [arXiv:2007.06594]

Villanova, S., Geisler, D., Carraro, G., Moni Bidin, C., \& Muñoz, C. 2013, ApJ, 778,186

Villanova, S., Monaco, L., Geisler, D., et al. 2019, ApJ, 882, 174

Wang, L., Kroupa, P., Takahashi, K., \& Jerabkova, T. 2020, MNRAS, 491, 440

White, S. D. M., \& Rees, M. J. 1978, MNRAS, 183, 341

\section{Appendix A: Na abundances of Milky Way globular clusters}
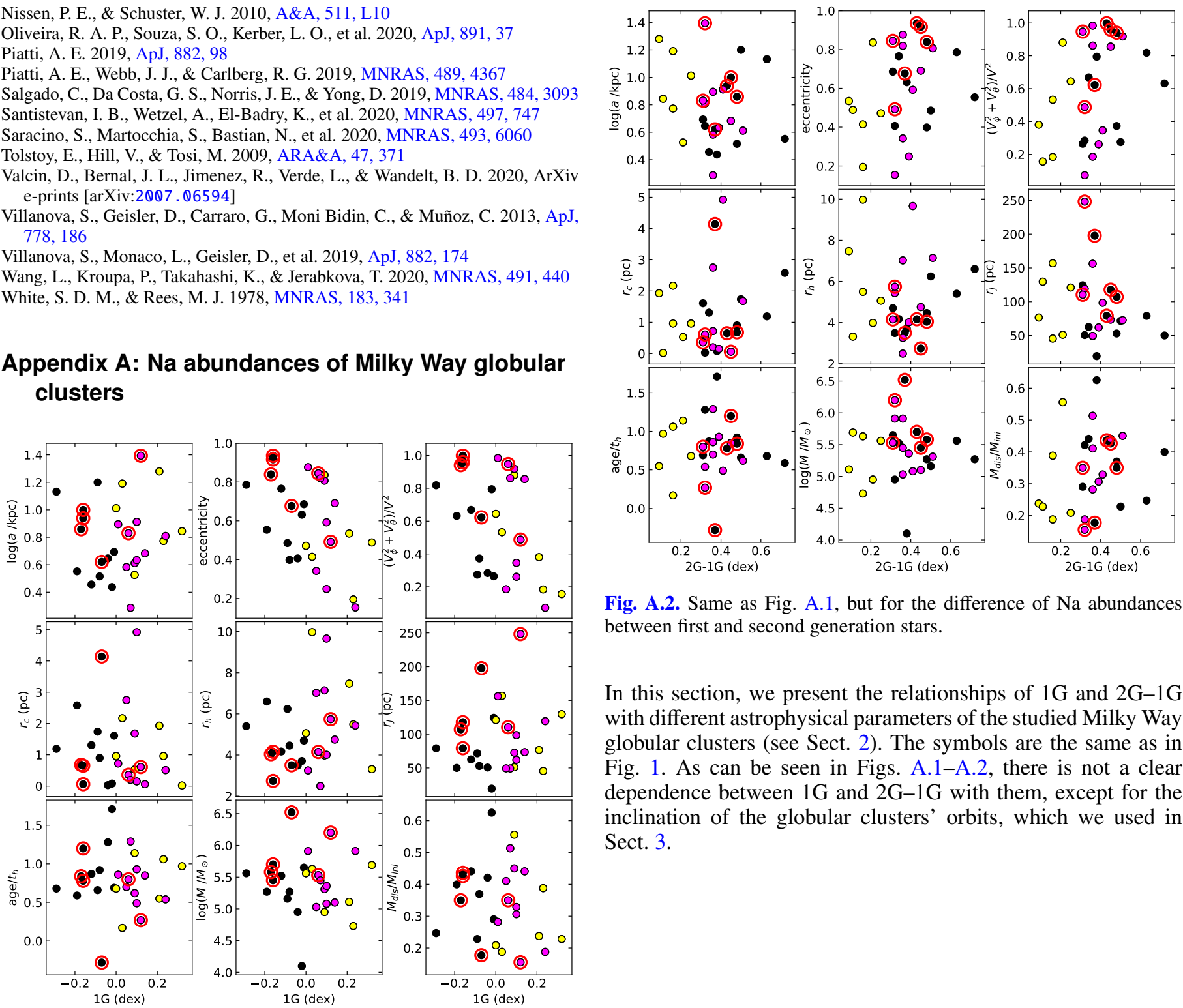

Fig. A.2. Same as Fig. A.1, but for the difference of $\mathrm{Na}$ abundances between first and second generation stars.

In this section, we present the relationships of $1 \mathrm{G}$ and $2 \mathrm{G}-1 \mathrm{G}$ with different astrophysical parameters of the studied Milky Way globular clusters (see Sect. 2). The symbols are the same as in Fig. 1. As can be seen in Figs. A.1-A.2, there is not a clear dependence between $1 \mathrm{G}$ and $2 \mathrm{G}-1 \mathrm{G}$ with them, except for the inclination of the globular clusters' orbits, which we used in Sect. 3.

Fig. A.1. Na abundances of first generation stars as a function of different globular clusters' parameters, namely: semi-major axis $(a)$ of the globular cluster orbits, the eccentricity of the globular cluster orbits, space velocity components $V_{\phi}$ and $V_{\theta}, V^{2}=V_{\phi}^{2}+V_{\theta}^{2}+V_{r}^{2}$, core radius $\left(r_{c}\right)$, half-mass radius $\left(r_{h}\right)$, Jacobi radius $\left(r_{J}\right)$, age to half-mass relaxation time ratio (age $/ t_{h}$ ), globular cluster mass, and ratio of the mass lost by tidal disruption to the total globular cluster mass $\left(M_{\mathrm{dis}} / M_{\mathrm{ini}}\right)$. 